\title{
Problems of Fuzzy c-Means and similar algorithms with high dimensional data sets
}

Roland Winkler (roland.winkler@dlr.de), Frank Klawonn, Rudolf Kruse July 20, 2010 


\section{Outline}

(1) FCM (and similar) Algorithms in high dimensions

(2) High Dimension Test Environment

(3) Effects of high dimensional data sets

(4) Conclusions

(5) Literature

(6) Appendix 


\section{Current Section}

(1) FCM (and similar) Algorithms in high dimensions

(2) High Dimension Test Environment

(3) Effects of high dimensional data sets

(4) Conclusions

(5) Literature

(6) Appendix 


\section{Prototype based algorithms}

- Objective function that needs to be minimized:

$$
J=\sum_{i=1}^{c} \sum_{j=1}^{n} f\left(u_{i j}\right) d_{i j}^{2}
$$

- c: number of prototypes, $n$ : number of data objects, $u_{i j}$ membership value, $f: \mathbb{R} \rightarrow \mathbb{R}$ : fuzzifier function, $d_{i j}=\left\|y_{i}-x_{j}\right\|, y_{i}$ : prototype, $x_{j}$ : data object, $1=\sum_{i=1}^{c} u_{i j}$

- artificial generated data set 1: 2 dimensions, 4 clusters, 1000 data objects per cluster, $10 \%$ noise

- artificial generated data set 2: 50 dimensions, 100 clusters, 100 data objects per cluster, $10 \%$ noise 


\section{$\mathrm{HCM}$}
- $f(u)=u$

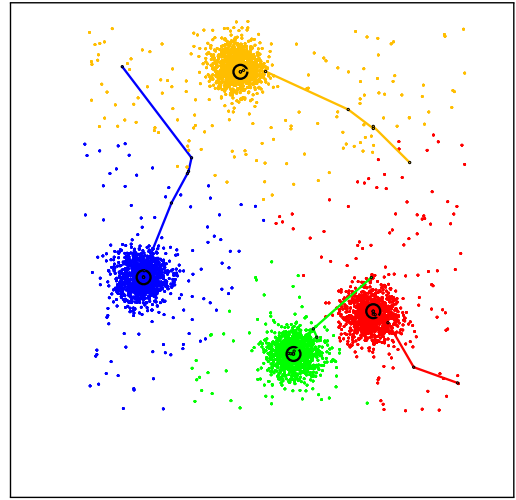

Data set 1

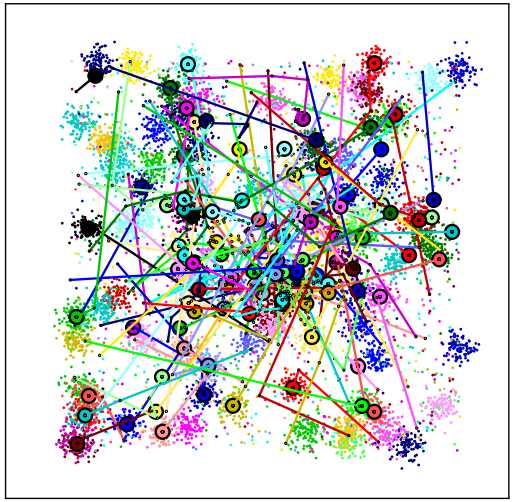

Data set 2, found clusters: 40 


\section{FCM}

- $f(u)=u^{\omega}, \omega=2$

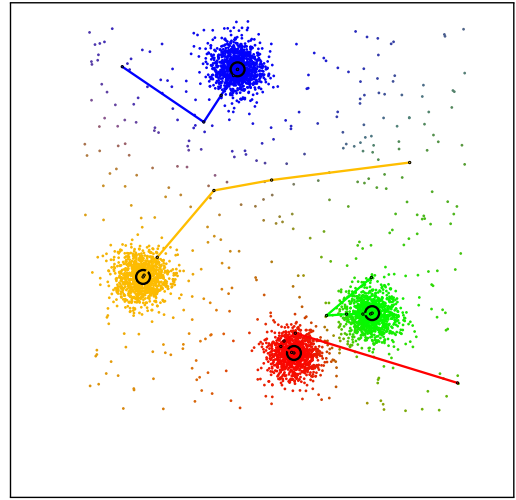

Data set 1

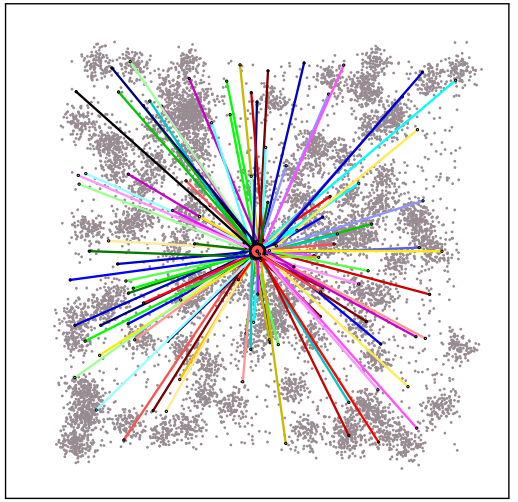

Data set 2, found clusters: 0 


\section{NFCM}

- $f(u)=u^{\omega}, \omega=2$, noise cluster at $d_{\text {noise }}=0.3$

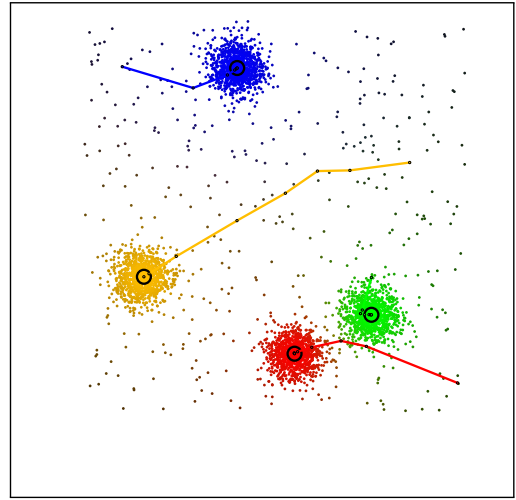

Data set 1

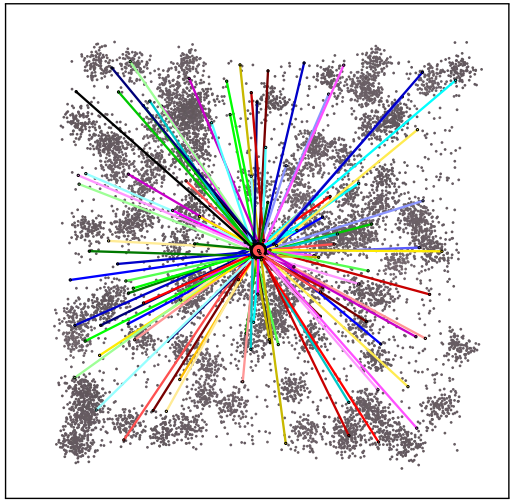

Data set 2, found clusters: 0 


\section{PFCM}

- $f(u)=\left(\frac{1-\beta}{1+\beta} u^{2}+\frac{2 \beta}{1+\beta} u\right), \beta=0.5$

- in a 2 cluster environment: $u_{1 j}=1 \Leftrightarrow \frac{d_{1 j}}{d_{2 j}}<\beta$

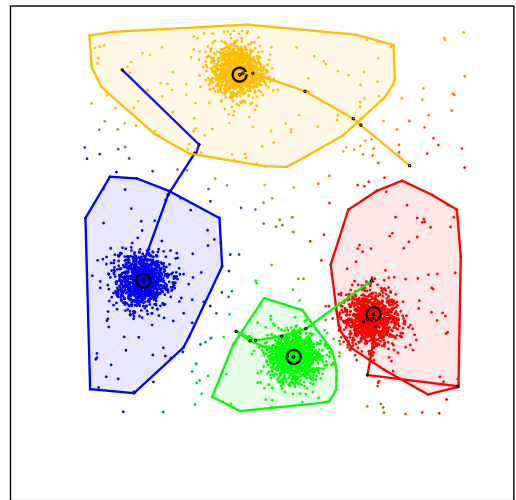

Data set 1

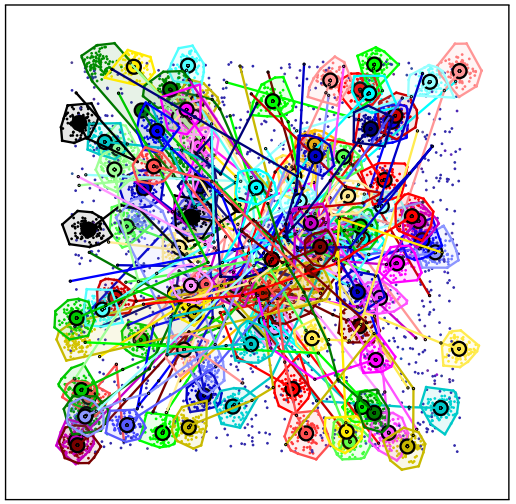

Data set 2, found clusters: 90

\section{Deutsches Zentrum}

in der Helmholtz-Gemeinschaft 


\section{PNFCM}

$$
\text { - } f(u)=\left(\frac{1-\beta}{1+\beta} u^{2}+\frac{2 \beta}{1+\beta} u\right), \beta=0.5, d_{\text {noise }}=0.3
$$

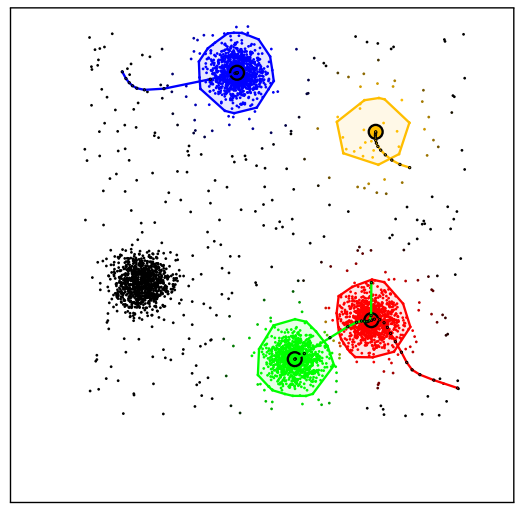

Data set 1

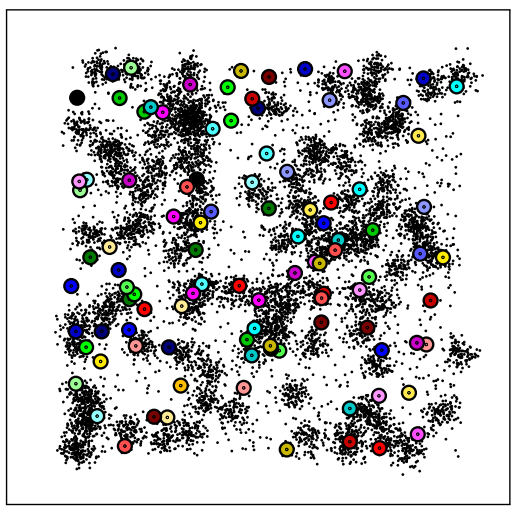

Data set 2, found clusters: 0

Deutsches Zentrum 


\title{
Current Section
}

\section{(1) FCM (and similar) Algorithms in high dimensions}

\author{
(2) High Dimension Test Environment
}

(3) Effects of high dimensional data sets

(4) Conclusions

(5) Literature

(6) Appendix 


\section{Test setup}

- Generate a data set with $n=c$ data objects representing $c$ clusters of infinite density

- Place the data objects on a $D$-dimensional hypersphere surface of radios 1 such that the pairwise distances are maximised

- Place prototypes in the centre of gravity (cog) and gradually move them to the data objects

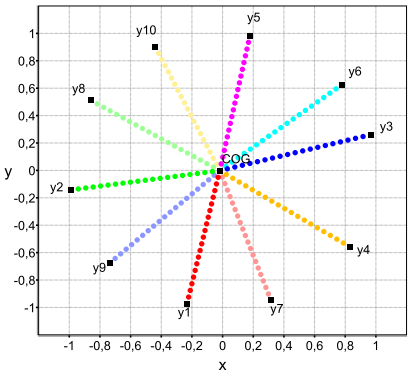

- Observe the objective function 


\section{Tests}

- Applying the algorithms

- $y_{i}=(1-\alpha) \cdot \operatorname{cog}+\alpha x_{i}$ for $\alpha \in[0,1]$

- Normalise the objective function by the value of the objective function at $\alpha=0$

- Let $\alpha$ gradually increase from 0 to 1 and monitor the normalized objective function value

- 2 Test setups

- Setup 1: 100 prototypes, between 2 and 200 dimensions

- Setup 2: 50 dimensions, between 2 and 500 prototypes 


\section{Current Section}

\section{(1) FCM (and similar) Algorithms in high dimensions}

(2) High Dimension Test Environment

(3) Effects of high dimensional data sets

(4) Conclusions

(5) Literature

(6) Appendix 


\section{Objective function of $\mathrm{HCM}$}

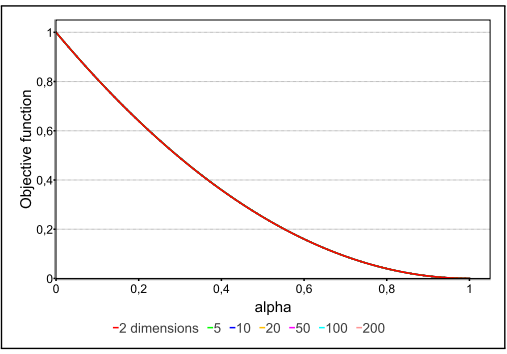

100 prototypes various dimensions

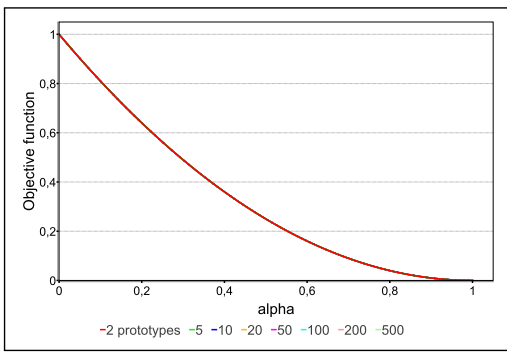

50 Dimensions various prototypes 


\section{Objective function of FCM}

- $f(u)=u^{\omega}, \omega=2$

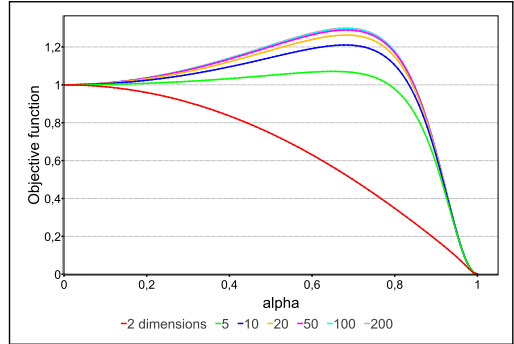

100 prototypes various dimensions

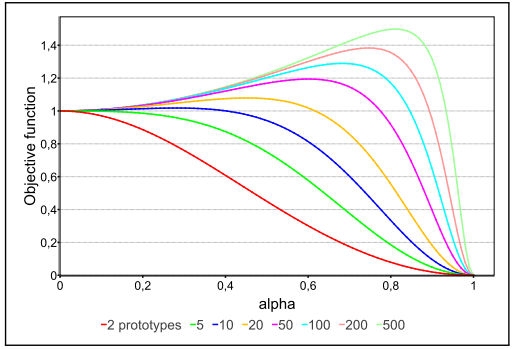

50 Dimensions various prototypes 


\section{Objective function of NFCM}

- $f(u)=u^{\omega}, \omega=2, d_{\text {noise }}=\frac{1}{2}$

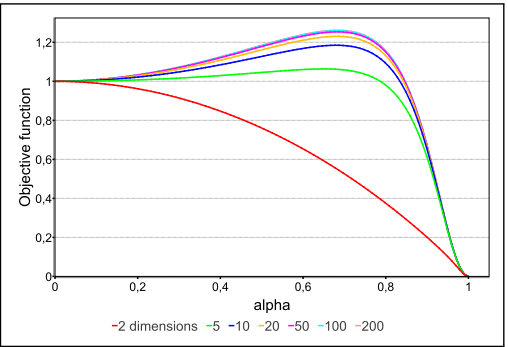

100 prototypes various dimensions

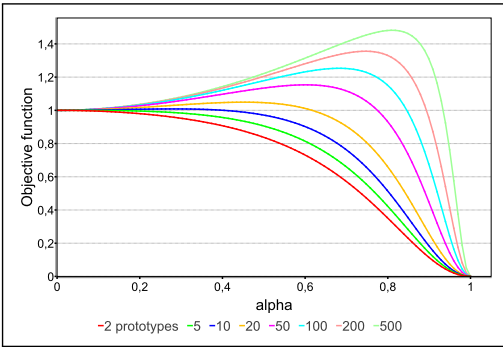

50 Dimensions various prototypes 


\section{Objective function of PFCM}

$$
\text { - } f(u)=\left(\frac{1-\beta}{1+\beta} u^{2}+\frac{2 \beta}{1+\beta} u\right), \beta=\frac{1}{2}
$$

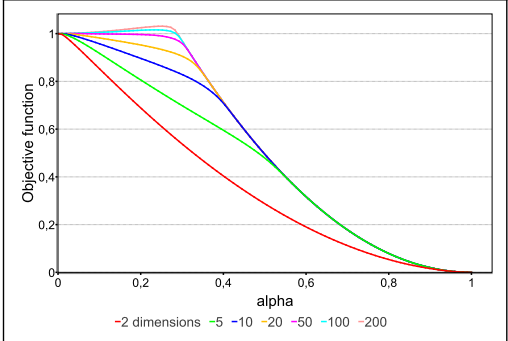

100 prototypes various dimensions

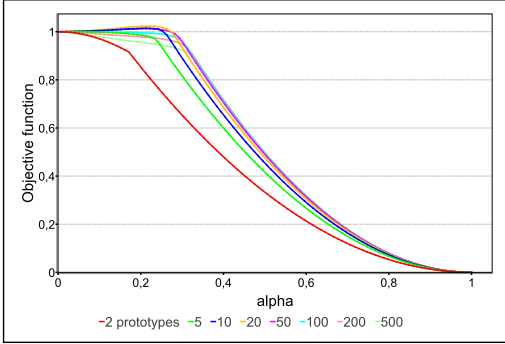

50 Dimensions various prototypes 


\section{Objective function of PNFCM}

- $f(u)=\left(\frac{1-\beta}{1+\beta} u^{2}+\frac{2 \beta}{1+\beta} u\right), \beta=\frac{1}{2}, d_{\text {noise }}=\frac{1}{2}$

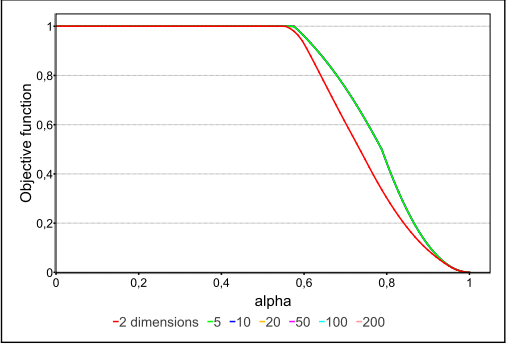

100 prototypes

various dimensions

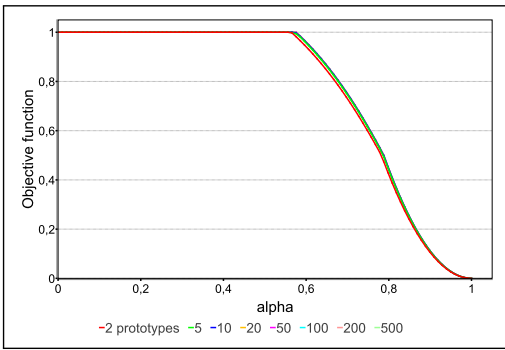

50 Dimensions various prototypes 


\section{Tweak the fuzzy based algorithms to work at high dimensions}

- For FCM, a dimension dependent fuzzifier can be used to counter the dimension effect: $\omega=1+\frac{2}{D}$

- With a tweaked fuzzifier, NFCM behaves like PNFCM and needs a dimension dependent noise distance: $d_{\text {noise }}=0.5 \log _{2}(D)$

- PNFCM requires also requires an dimension dependent noise distance: $d_{\text {noise }}=0.5 \log _{2}(D)$ 


\section{FCM with adjusted parameters}

- $f(u)=u^{\omega}, \omega=1+\frac{2}{D}$

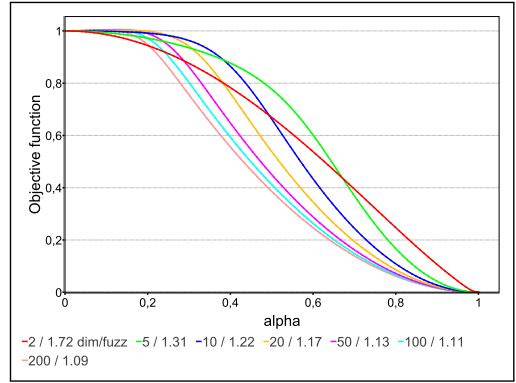

\section{0 prototypes} various dimensions

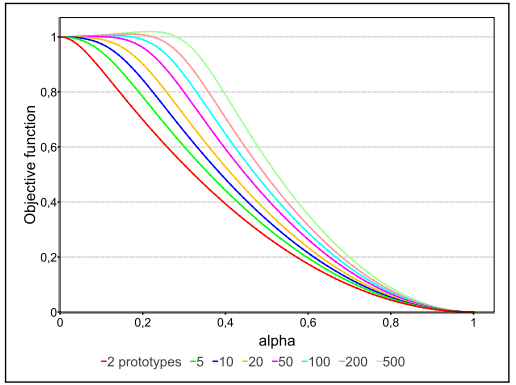

50 Dimensions various prototypes 


\section{FCM with adjusted parameters}

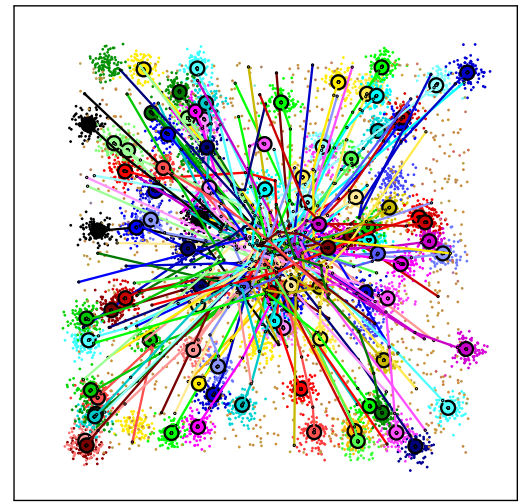

50 Dimensions, 100 clusters, found clusters: 92 


\section{NFCM with adjusted parameters}

- $f(u)=u^{\omega}, \omega=1+\frac{2}{D}, d_{\text {noise }}=0.5 \log _{2}(D)$

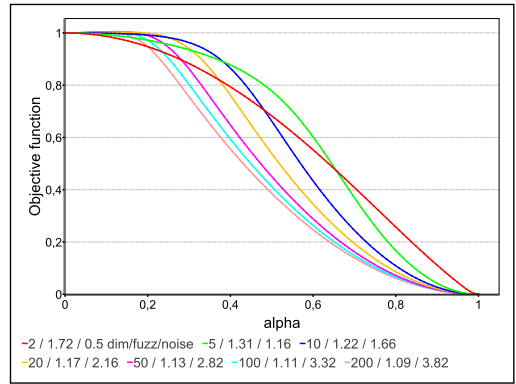

\section{0 prototypes} various dimensions

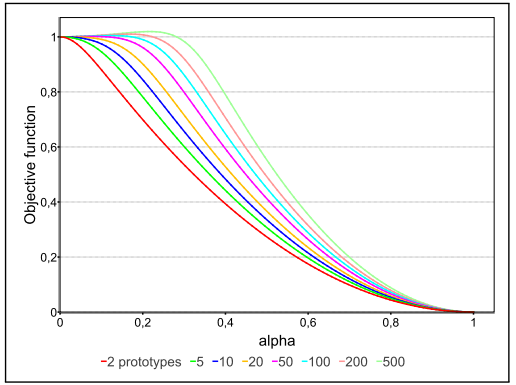

50 Dimensions various prototypes 


\section{NFCM with adjusted parameters}

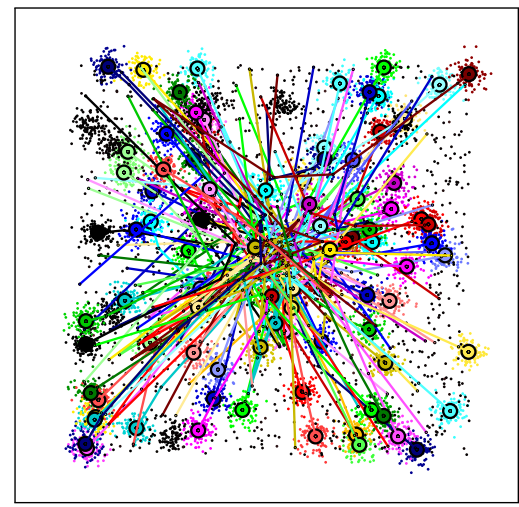

50 Dimensions, 100 clusters, found clusters: 91 , correct clustered noise: 1000 , incorrect clustered as noise: 900 


\section{PNFCM with adjusted parameters}

$$
\text { - } f(u)=\left(\frac{1-\beta}{1+\beta} u^{2}+\frac{2 \beta}{1+\beta} u\right), \beta=0.5, d_{\text {noise }}=0.5 \log _{2}(D)
$$

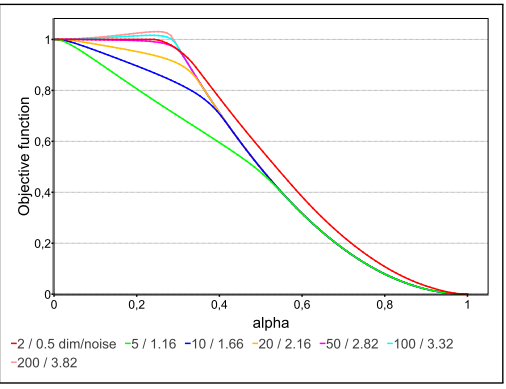

100 prototypes various dimensions

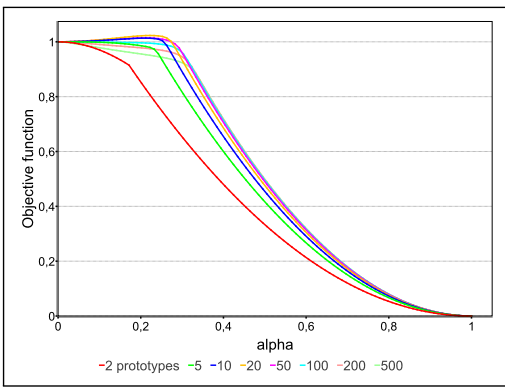

50 Dimensions various prototypes 


\section{PNFCM with adjusted parameters}

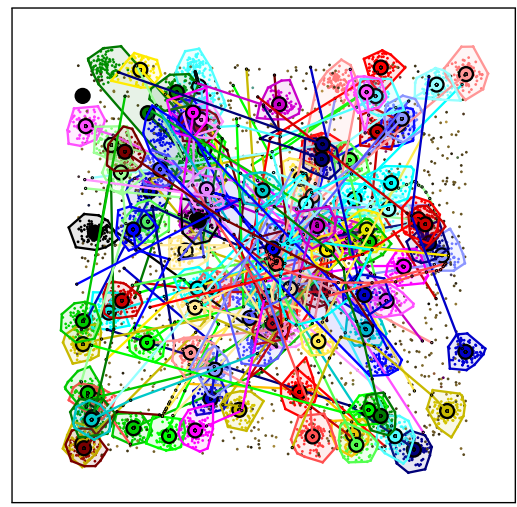

50 Dimensions, 100 clusters, found clusters: 90 , correct clustered noise: 991 , incorrect clustered as noise: 0 


\section{Current Section}

\section{(1) FCM (and similar) Algorithms in high dimensions}

(2) High Dimension Test Environment

(3) Effects of high dimensional data sets

\section{(4) Conclusions}

(5) Literature

(6) Appendix 


\section{Scoring the algorithms}

- 50 Dimensions, 100 cluster with 100 data objects each, 1000 noise data objects

\begin{tabular}{|l|lll|}
\hline Algorithm & Found clusters & $\begin{array}{l}\text { Correctly } \\
\text { clustered noise }\end{array}$ & $\begin{array}{l}\text { Incorrect } \\
\text { clustered as } \\
\text { noise }\end{array}$ \\
\hline HCM & 40 & 0 & 0 \\
FCM & 0 & 0 & 0 \\
NFCM & 0 & 1000 & 10000 \\
PFCM & 90 & 0 & 0 \\
PNFCM & 0 & 1000 & 10000 \\
\hline Adjusted & & & \\
Parameter & & 0 & 0 \\
\hline FCM AP & 92 & 1000 & 900 \\
NFCM AP & 91 & 994 & 0 \\
PNFCM AP & 90 & & \\
\hline
\end{tabular}




\section{Effects of high dimensions on the feature space}

- Let $S$ be a hypersphere of $D$ dimensions with radius $R$.

- $S$ has a volume of $V=C_{D} \cdot R^{D}$ with $C_{D}=\frac{\pi^{\frac{D}{2}}}{\Gamma\left(\frac{D}{2}+1\right)}$ only depending on $D$

- Let $S^{*}$ be a hypersphere of $D$ dimensions with radius $R^{*}$ and $V^{*}=C_{D} \cdot R^{* D}=\frac{1}{2} V$

- Then $R^{*}=\left(\frac{1}{2}\right)^{\frac{1}{D}} R$

- Example:

$$
\begin{array}{llll}
D=2: & R^{*}=0.70711 R & D=10: & R^{*}=0.93303 R \\
D=100: & R^{*}=0.99309 R & D=1000: & R^{*}=0.99931 R
\end{array}
$$




\section{Current Section}

\section{(1) FCM (and similar) Algorithms in high dimensions}

(2) High Dimension Test Environment

(3) Effects of high dimensional data sets

(4) Conclusions

(5) Literature

(6) Appendix 


\section{Literature}

㢄 Kevin Beyer, Jonathan Goldstein, Raghu Ramakrishnan, and Uri Shaft.

When is nearest neighbor meaningful?

In Database Theory - ICDT'99, volume 1540 of Lecture Notes in Computer Science, pages 217-235.

Springer Berlin / Heidelberg, 1999.

F. Höppner, F. Klawonn, R. Kruse, and T. Runkler.

Fuzzy Cluster Analysis.

John Wiley \& Sons, Chichester, England, 1999.

言

Frank Klawonn and Frank Höppner.

What is fuzzy about fuzzy clustering? understanding and improving the concept of the fuzzifier. In Cryptographic Hardware and Embedded Systems - CHES 2003, volume 2779 of Lecture Notes in Computer Science, pages 254-264. Springer Berlin / Heidelberg, 2003. 


\section{Thank you for your attention}




\section{Current Section}

\section{(1) FCM (and similar) Algorithms in high dimensions}

(2) High Dimension Test Environment

(3) Effects of high dimensional data sets

(4) Conclusions

(5) Literature

\section{(6) Appendix}




\section{Hard c-means (HCM)}

- Clustering Problem: data objects $X=\left\{x_{1}, \ldots, x_{n}\right\}$, prototypes $Y=\left\{y_{1}, \ldots, y_{c}\right\}$

- Objective Function: $\boldsymbol{J}_{\mathrm{HCM}}=\min _{S} \sum_{j=1}^{n} \sum_{j \in S_{i}} d_{i j}^{2}$

with $d_{i j}=\left\|y_{i}-x_{j}\right\|, S=\left\{S_{1}, \ldots, S_{c}\right\}, S_{i} \subset \mathbb{N}$ and $S_{i} \cap S_{k}=\emptyset$ for $i \neq k$.

- updated with:

$$
S_{i}=\left\{j: d_{i j} \leq d_{i k} \forall k\right\} \quad \text { and } y_{i}=\frac{1}{\left|S_{i}\right|} \sum_{j \in S_{i}} x_{j}
$$




\section{HCM membership values example}

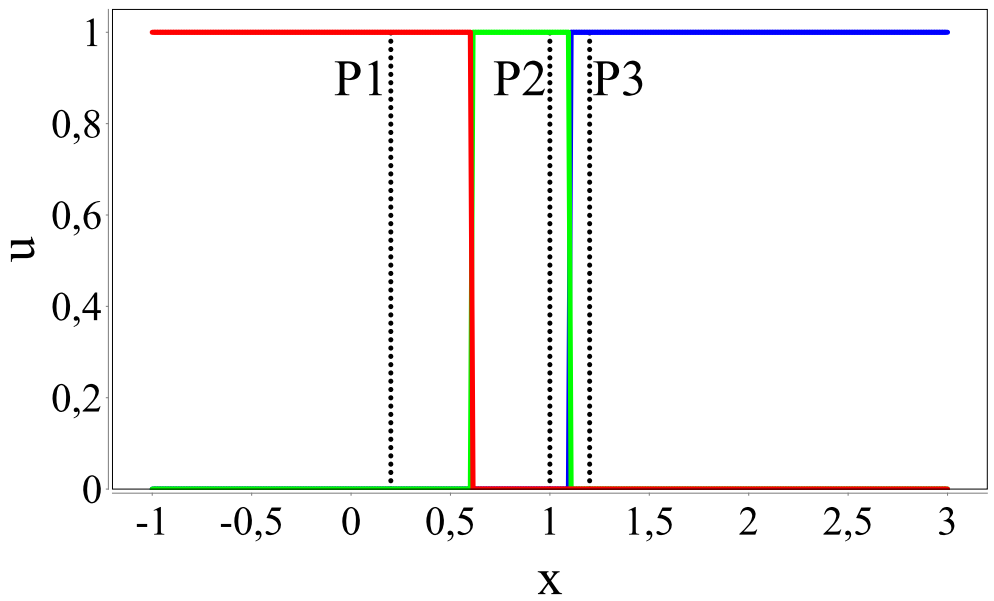




\section{Fuzzy c-means (FCM)}

- Clustering Problem: data objects $X=\left\{x_{1}, \ldots, x_{n}\right\}$, prototypes $Y=\left\{y_{1}, \ldots, y_{c}\right\}$

- Objective Function: $\boldsymbol{J}_{\mathrm{FCM}}=\sum_{i=1}^{c} \sum_{j=1}^{n} u_{i j}^{\omega} d_{i j}^{2}$

$$
\text { with } d_{i j}=\left\|y_{i}-x_{j}\right\|, 1=\sum_{i=1}^{c} u_{i j}, u_{i j}>1 \text { and } \omega>1 \text {. }
$$

- updated with:

$$
u_{i j}=\frac{d_{i j}^{\frac{2}{1-\omega}}}{\sum_{k=1}^{c} d_{k j}^{\frac{2}{1-\omega}}} \text { and } y_{i}=\frac{\sum_{j=1}^{n} u_{i j}^{\omega} \cdot x_{j}}{\sum_{j=1}^{n} u_{i j}^{\omega}} \text {. }
$$




\section{FCM fuzzified membership values example}

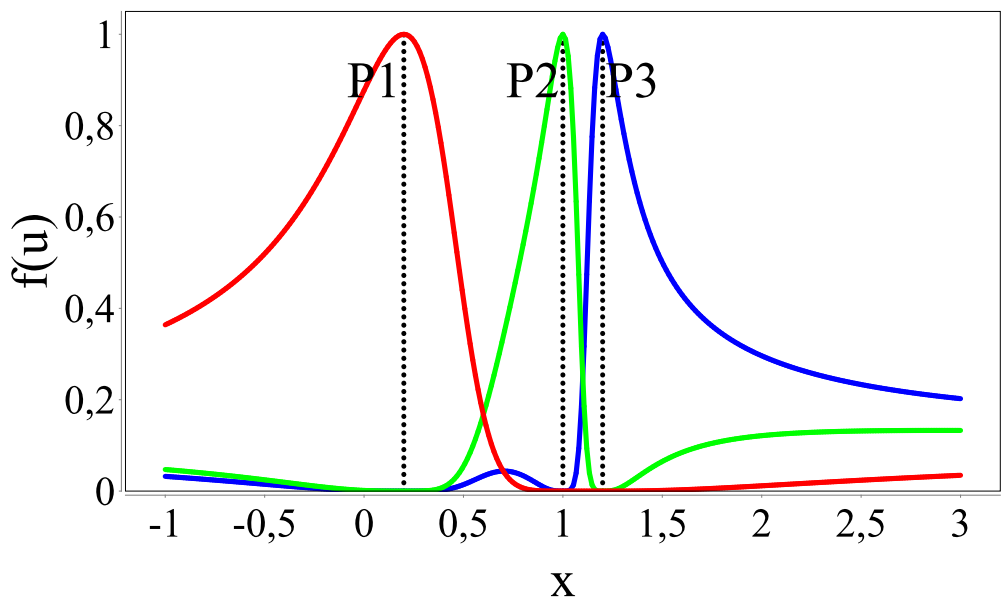




\section{Noise Fuzzy c-means (NFCM)}

- Objective Function: $\boldsymbol{J}_{\mathrm{NFCM}}=\sum_{i=0}^{c} \sum_{j=1}^{n} u_{i j}^{\omega} d_{i j}^{2}$ with $d_{0 j}=d_{\text {noise }} \geq 0, d_{i j}=\left\|y_{i}-x_{j}\right\|$ for $i=1 \ldots c$, $1=\sum_{i=1}^{c} u_{i j}, u_{i j}>1$ and $\omega>1$.

- updated with:

$$
u_{i j}=\frac{d_{i j}^{\frac{2}{1-\omega}}}{\sum_{k=0}^{c} d_{k j}^{\frac{2}{1-\omega}}} \text { and } y_{i} \stackrel{i=1 \ldots c}{=} \frac{\sum_{j=1}^{n} u_{i j}^{\omega} \cdot x_{j}}{\sum_{j=1}^{n} u_{i j}^{\omega}}
$$




\section{NFCM fuzzified membership values example}

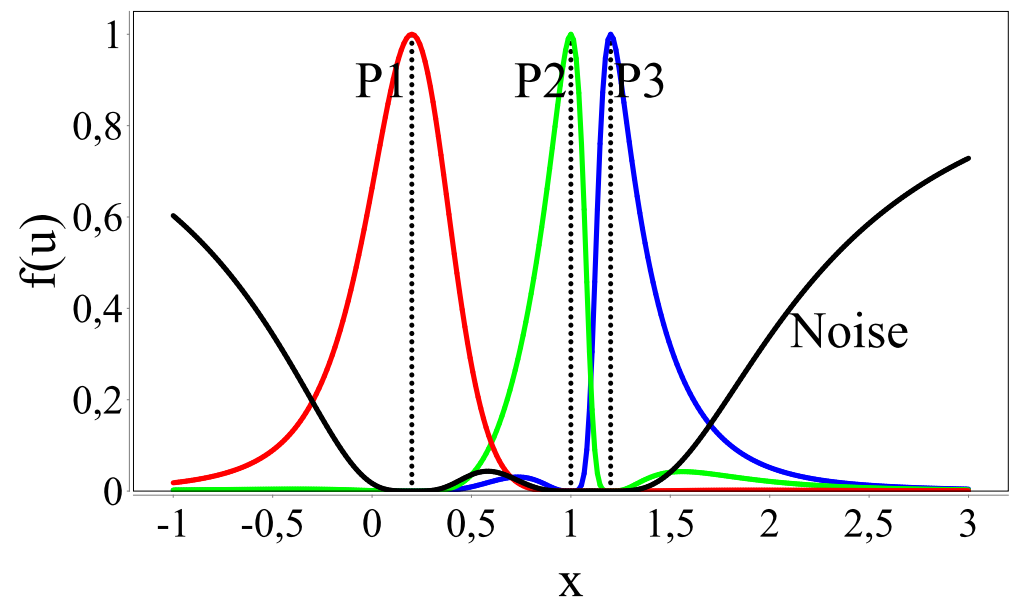




\section{Fuzzy c-means with polynomial fuzzifier (PFCM)}

- $\boldsymbol{J}_{\mathrm{PFCM}}=\sum_{i=1}^{c} \sum_{j=1}^{n}\left(\frac{1-\beta}{1+\beta} u_{i j}^{2}+\frac{2 \beta}{1+\beta} u_{i j}\right) d_{i j}^{2}$ with $d_{i j}=\left\|y_{i}-x_{j}\right\|$, $1=\sum_{i=1}^{c} u_{i j}, u_{i j}>1$ and $0 \geq \beta \geq 1$.

- with $\varphi$ is a permutation that sorts the prototypes ascending by their distance and $\hat{c}$ is the number of clusters that have a positive membership value:

$$
u_{i j}= \begin{cases}\frac{1}{1-\beta}\left(\frac{1+(\hat{c}-1) \beta}{\sum_{k=1}^{\hat{c}} \frac{d_{i j}^{2}}{d_{\varphi(k) j}^{2}}}-\beta\right) & \text { iff } \varphi(i) \leq \hat{c} \\ 0 & \text { otherwise }\end{cases}
$$

- in a 2 cluster environment: $u_{1 j}=1 \Leftrightarrow \frac{d_{1 j}}{d_{2 j}}<\beta$ 


\section{PFCM fuzzified membership values example}

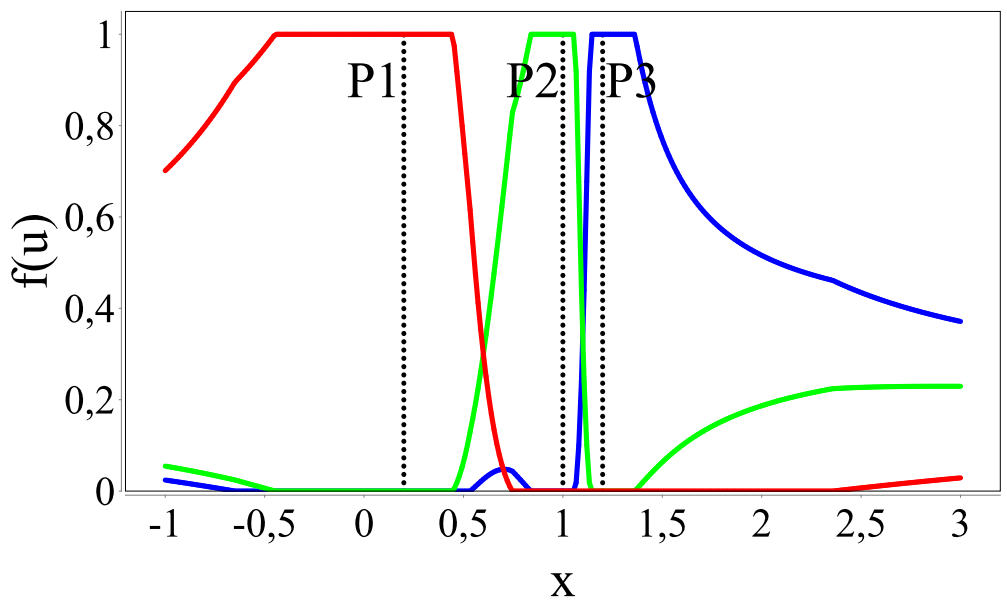




\section{Noise fuzzy c-means with polynomial fuzzifier (PNFCM) [3]}

- $\boldsymbol{J}_{\mathrm{PNFCM}}=\sum_{i=0}^{c} \sum_{j=1}^{n}\left(\frac{1-\beta}{1+\beta} u_{i j}^{2}+\frac{2 \beta}{1+\beta} u_{i j}\right) d_{i j}^{2}$ with

$$
\begin{aligned}
& d_{0 j}=d_{\text {noise }} \geq 0, d_{i j}=\left\|y_{i}-x_{j}\right\| \text { for } i=1 \ldots c, 1=\sum_{i=1}^{c} u_{i j}, \\
& u_{i j}>1 \text { and } 0 \geq \beta \geq 1 .
\end{aligned}
$$

- with $\varphi$ and $\hat{c}$ as for PFCM:

$$
u_{i j}= \begin{cases}\frac{1}{1-\beta}\left(\frac{1+(\hat{c}-1) \beta}{\sum_{k=0}^{\hat{c}} \frac{d_{i j}^{2}}{d_{\varphi(k) j}^{2}}}-\beta\right) & \text { iff } \quad \varphi(i) \leq \hat{c} \\ 0 & \text { otherwise }\end{cases}
$$




\section{PNFCM fuzzified membership values example}

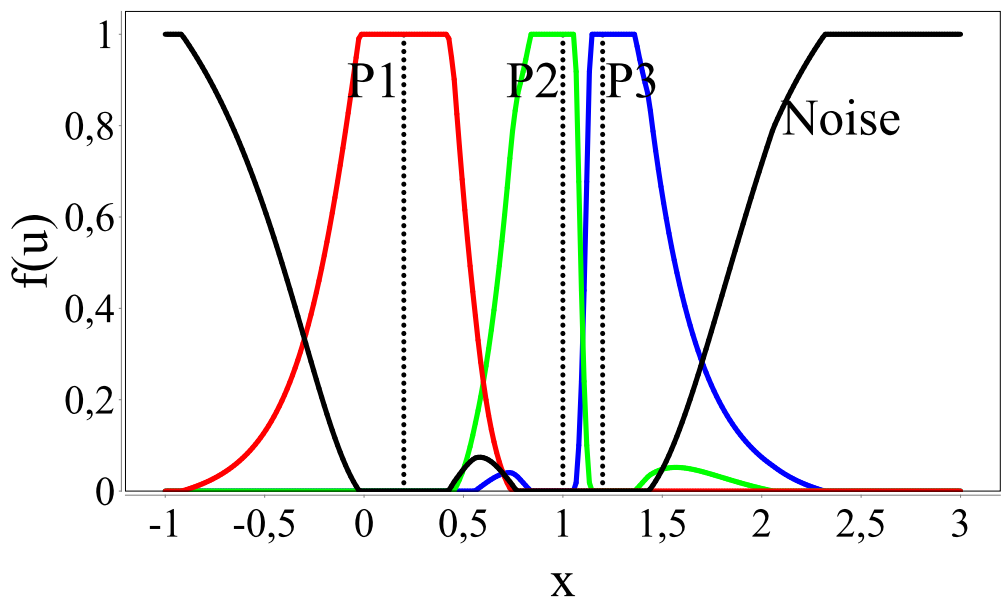




\section{Maximal separated data objects on a hypersphere surface}

- Sample many $D$-dimensional normal distributed data objects and project them to length 1

- The resulting data objects follow a rotation symmetric probability distribution

- Run Hard k-Means on the random generated data set

- Project prototypes on the hypersphere surface as new data objects 


\section{Data set properties}

\begin{tabular}{|c|c|c||c|c|c|}
\hline Dim & Clusters & $\begin{array}{c}\text { Data } \\
\text { Objects }\end{array}$ & $\begin{array}{c}\text { per Cluster } \\
\text { (Min - Max) }\end{array}$ & $\begin{array}{c}\text { Angle }\left[{ }^{\circ}\right] \\
\text { (Min) }\end{array}$ & $\begin{array}{c}\text { Distance } \\
\text { (Min }- \text { Max })\end{array}$ \\
\hline 2 & 100 & 100000 & $811-3256$ & 1.46 & $0.0253-2.0$ \\
5 & 100 & 100000 & $1800-2235$ & 38.8 & $0.665-1.99$ \\
10 & 100 & 100000 & $1795-2237$ & 54.1 & $0.910-1.95$ \\
20 & 100 & 100000 & $1831-2151$ & 60.9 & $1.01-1.84$ \\
50 & 100 & 100000 & $1826-2162$ & 74.0 & $1.20-1.74$ \\
100 & 100 & 100000 & $1853-2137$ & 77.3 & $1.25-1.63$ \\
\hline 50 & 50 & 100000 & $3772-4192$ & 80.3 & $1.29-1.60$ \\
50 & 100 & 100000 & $1839-2120$ & 72.5 & $1.18-1.72$ \\
50 & 200 & 100000 & $901-1114$ & 70.0 & $1.15-1.75$ \\
50 & 500 & 100000 & $309-480$ & 63.0 & $1.04-1.77$ \\
\hline
\end{tabular}

